\title{
Sectorial optic atrophy and homonymous, horizontal sectoranopia: a lateral choroidal artery syndrome?
}

\author{
L.FR ISÉN, L. H O L M E G A R D, A N D M. R OSENCRANTZ
}

From the Department of Ophthalmology and the Section of Neuroradiology, University of Göteborg, Sweden

SUMMARY Two patients with sectorial optic atrophy and homonymous, horizontal sectoranopia are described. Neuroradiological investigations localised the visual pathway lesion to the lateral geniculate body. The peculiar nature of the field defect and the optic atrophy appeared explicable in terms of ischaemia in the territory of the lateral choroidal artery.

An incomplete interruption of the retrochiasmatic visual pathway sometimes results in loss of vision within homonymous and congruous, horizontally oriented wedges of the visual field. In isolation, such a horizontal sectoranopia has a debatable localising value. Spalding (1952) described this type of field defect in four cases with penetrating head injuries, and considered the field defect compatible with an incomplete lesion of the anterior part of the visual radiation. Smith (1970) described a patient with such a field cut of presumably vascular origin. Three cases with similar but somewhat incongruous field defects have been contributed by Hoyt (1975). One had a necropsy-proved astrocytoma involving the lateral geniculate body. The other two patients presented a peculiar form of optic atrophy, so-called homonymous, hemioptic atrophy, that is characteristic of a retrochiasmatic lesion of the anterior visual pathway. The precise nature and location of their lesions were not known. Both had signs of vascular disease.

We describe here two patients with homonymous and congruous horizontal sectoranopia, and corresponding optic atrophy, where neuroradiological investigations localised the lesion to the lateral geniculate nucleus (LGN). Our findings were further analysed in the light of recent advances in the understanding of the representation of the visual field in the LGN. It appears that the syndrome is explicable in terms of damage to that

Address for reprint requests : Dr L. Frisén, Ögonkliniken, Sahlgrenska sjukhuset, S-413 45 Göteborg, Sweden.

Accepted 4 November 1977 part of the LGN supplied by the lateral choroidal artery, with sparing of those parts that derive their supply from the anterior choroidal artery.

\section{Case reports}

CASE 1

A 33 year old woman from southern Europe had suffered from chronic, nonspecific headaches for many years. There were no accompanying symptoms. Intermittent exacerbations had prompted the patient to attend the Emergency Unit of the Sahlgrenska University Hospital on several occasions. General medical and neurological examinations had never revealed anything abnormal.

On 21 March 1973, when preparing to unlock a door, she suddenly experienced black spots in her visual field, and was unable to find the keyhole by sight. Fifteen minutes later, severe frontal headache started, and she vomited once. When seen at the Emergency Unit a few hours later, she was in obvious distress and complained of dim vision. Nothing abnormal was found on medical examination except questionable nuchal rigidity.

On the next day confrontation tests revealed a left homonymous field defect, more pronounced in the lower quadrants. There were no other abnormal neurological signs. A lumbar tap produced grossly haemorrhagic cerebrospinal fluid with nonspecific spectrophotometric extinction. Right and left carotid angiograms and a vertebral angiogram were obtained. These were read as normal. No specific diagnosis was made. 
The patient was referred for neuro-opthalmological evaluation several months later. No new symptoms had appeared during this interval; the headaches had reverted to their habitual level. Abnormal findings were limited to the retinal nerve fibre layer in both eyes, and the visual fields (Figs 1 and 2). There was no clear-cut dicc pallor. There was loss of retinal nerve fibres in the papillomacular and nasal areas of the left fundus, depriving a nearly horizontal strip of the nasal hemiretina from communication with higher visual centres. The same sectors of the peripa- pillary area were normal in the right eye, but the arcuate bundles were conspicuously thinner than those in the left eye, as expected with descending atrophy involving the temporal hemiretina (Hoyt and Kommerell, 1973; Hoyt, 1975, 1976). This, then, was the picture of acquired, incomplete optic atrophy due to partial damage of second order visual pathway neurones somewhere behind the chiasm, on the right side. This analysis was corroborated by the finding of a left homonymous and congruous sectoranopia. The abruptly sloping borders of the field cut suggested a vascular
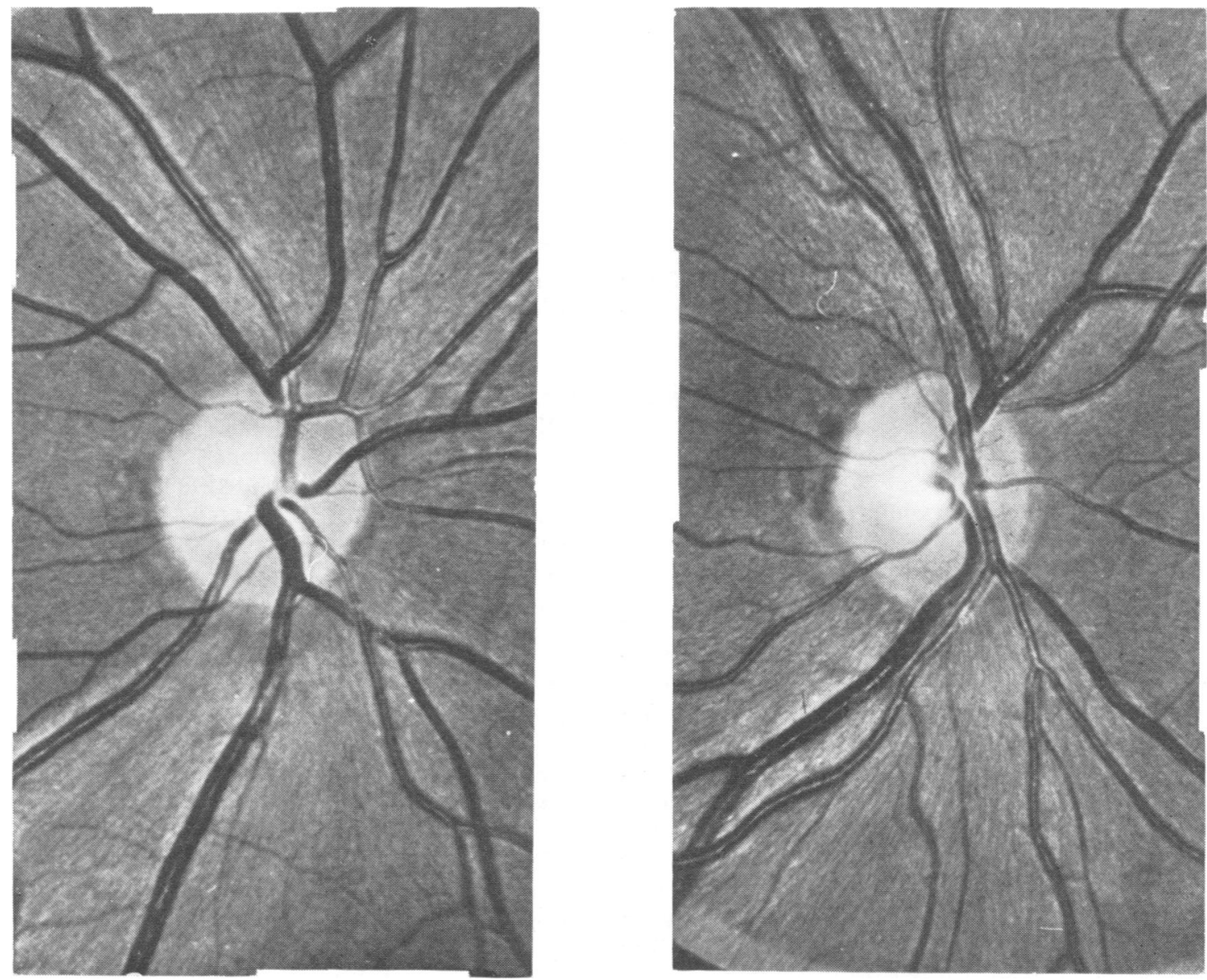

Fig. 1 Case 1: fundus photographs $(20 \times)$ demonstrating sectorial atrophy of the retinal nerve fibre layer. Atrophic sectors are indicated by notches in picture margins. There is loss of nerve fibre bundles in the nasal and papillomacular areas of the left eye (right photograph), causing failure of conduction from a nearly horizontal strip of the nasal hemiretina. Loss of nerve fibre bundles is evidenced by lack of fine, grey lines radiating from the optic disc, with consequent exposure of small epiretinal vessels. The arcuate bundles appear normal. Nasal and papillomacular sectors appear normal in the right eye (left photograph), but there is thinning of the arcuate bundles, particularly within their limiting sectors. This sign suggests defective conduction from the temporal hemiretina. 


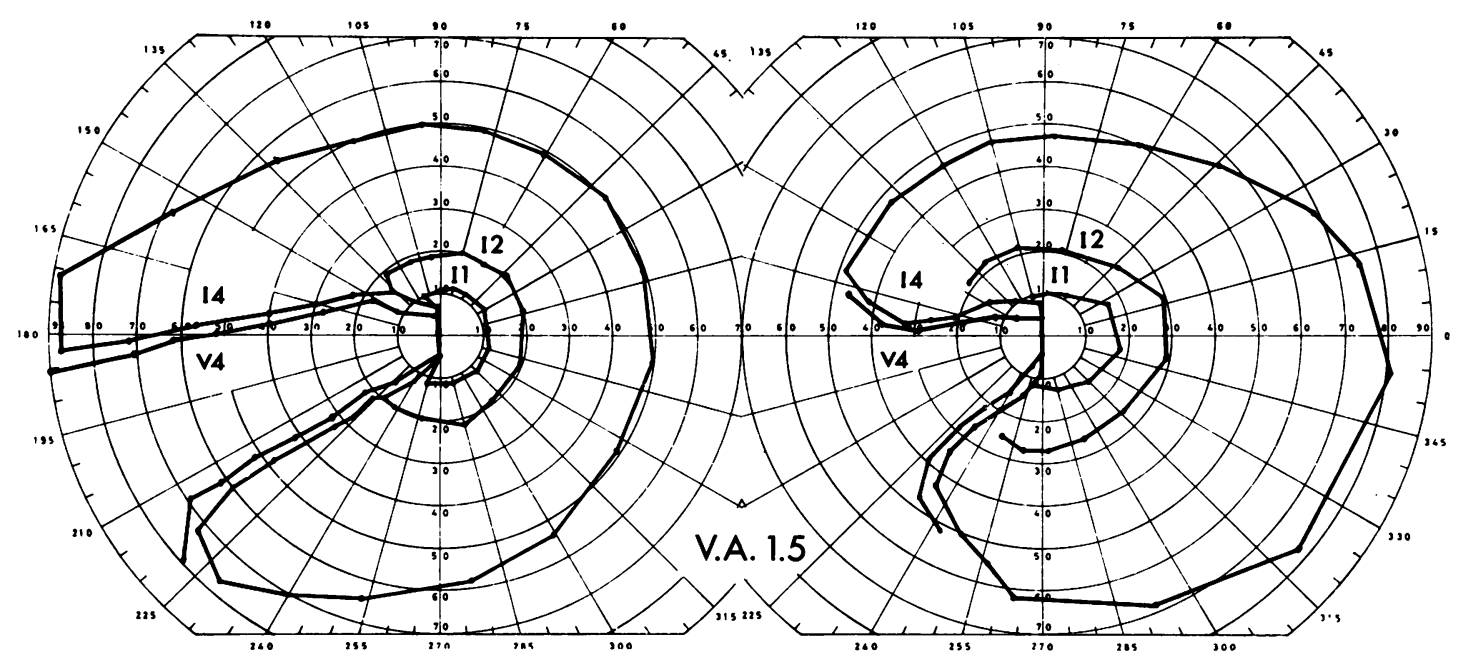

Fig. 2 Case 1: visual field defects.

lesion. These findings prompted review of the angiograms, with successful identification of the responsible lesion: a small vascular malformation of the anterior and lateral choroidal arteries on the right, in the region of the LGN (Fig. 3).

There were no changes at follow-up four years later.

\section{CASE 2}

A male mechanic had suffered from vertiginous attacks and impaired hearing with tinnitus on the right since 1950 , when he was 20 years old. His symptoms gradually became more troublesome over the years, but clinical findings were limited to impaired hearing and somewhat poor caloric reactions on the right. An incomplete air study made in 1973 revealed a questionable dislocation of the fourth ventricle towards the left. A subsequent angiogram suggested downward displacement of the posterior inferior cerebellar artery on the right. Repeated brain scans were normal. The patient was referred to the Sahlgrenska University Hospital for neurosurgical exploration for a possible cerebellar mass on the right. The exploration met with completely normal conditions. The postoperative course was stormy, and the patient was re-explored twice on the suspicion of a postoperative haematoma. No haematoma was found, but a ventriculoperitoneal shunt was placed, and slow recovery ensued. There was also a remission of the vertiginous attacks.

One day in 1975, while watching television, the patient suddenly noted light spots moving around in his left peripheral visual field. The light spots were evanescent, but shortly afterwards the patient became aware of a visual field defect to the left, that subjectively remained unchanged. He did not seek medical aid until 1976 when the vertiginous attacks recurred.

Neurological, neuro-ophthalmological, and neuro-otological examinations were repeated several times during 1976 and 1977 , with virtually constant findings. The patient was noted to be somewhat slow and had an impaired memory. There was unspecific unsteadiness in the Romberg test, and right-beating jerk nystagmus. Optokinetic nystagmus was somewhat poorly sustained towards the right. Corneal sensitivity was symmetrical, but there were old keratitic scars and vascular shadows in the left cornea. Visual acuity was normal. There was an absolute, congruous, homonymous, horizontally oriented wedge-shaped visual field defect to the left, that failed to reach the vertical meridian (Fig. 4). Ophthalmoscopy and fundus photographs (not shown) revealed the same type of homonymous sectorial optic atrophy that was described in case 1 (Fig. 1).

Hearing tests suggested a cochlear lesion on the right. There was no clear-cut asymmetry upon caloric testing although there was striking directional preponderance towards the right.

Computerised tomography was carried out in 1977. Low degree hydrocephalus was revealed. The shunt catheter lay in the right posterior horn of the lateral ventricle. It reached down into the ambient cistern on the right, apparently having 

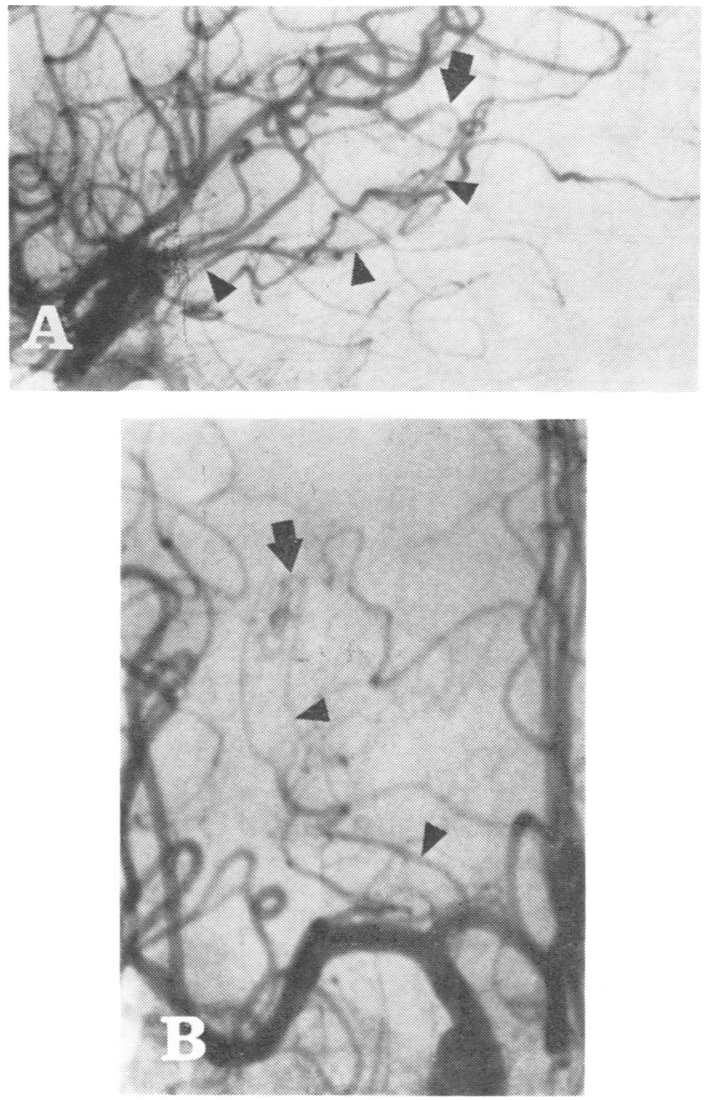

perforated the medial wall of the ventricle. There was a small rounded area of abnormally low attenuation in the region of the right LGN (Fig. 5). The corresponding $7 \times 7$ pixel array contained 33 pixels with attenuation values of 23 Hounsfield units, or less. The corresponding number on the left was 20 .

A unifying diagnosis was not obtained. Cogan's syndrome of nonsyphilitic interstitial keratitis with vestibuloauditory symptoms (Cogan, 1945) was considered, but no evidence was obtained of active collagen disease. The hearing problem was thought to be compatible with Ménière's disease but a central vestibular disorder was probably superimposed. The right LGN lesion was considered indicative of an old vascular lesion, possibly catheter-induced thrombosis of the lateral choroidal artery. This artery branches off the posterior cerebral artery in the ambient cistern, in the area of the tip of the ventricular catheter (see also below). Unfortunately, further investigations were refused.

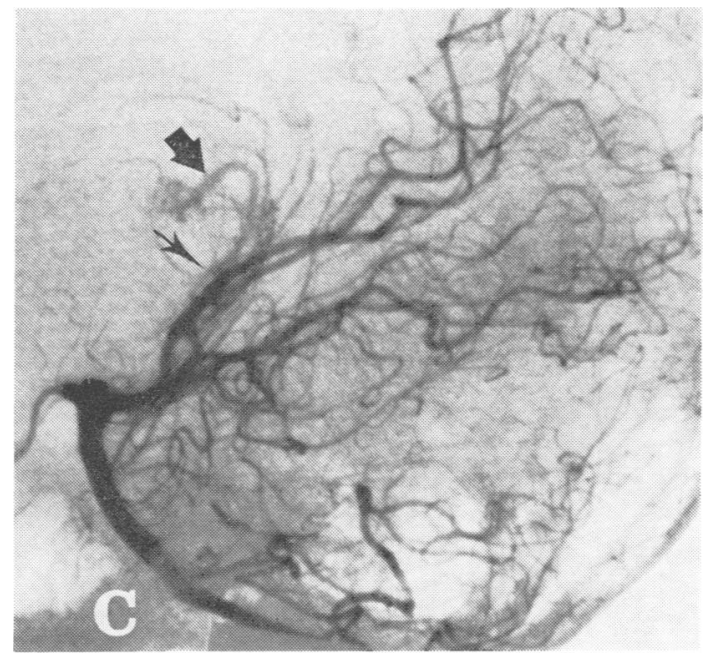

Fig. 3 Case 1: angiographic documentation of small vascular malformation (bold arrow) in the region of the right lateral geniculate body. Lateral $(A)$ and half axial projections $(B)$ of right internal carotid angiogram. Arrowheads identify the anterior choroidal artery. C. Vertebral angiogram in lateral view. Thin arrow identifies lateral choroidal artery.

\section{Discussion}

The lateral geniculate body, the site of the visual pathway lesion in our two patients, contains two nuclei: a smaller ventral nucleus of largely unknown importance (cf Spear et al., 1977), and a larger dorsal nucleus (LGN), where second order neurones of the visual pathway synapse with third order neurones. Because of its key position in the visual pathway, and its intricately layered structure, LGN has attracted much interest from morphologists and experimental physiologists, but so far little is known about functional tasks other than those of a relay station. It is only comparatively recently that detailed retinotopic maps have been obtained in experimental animals (see Malpeli and Baker (1975) for a recent review). What little is known about retinotopic organisation in man (Henschen, 1898; Mackenzie et al., 1933; Kupfer, 1962) appears to comply with that of the monkey Macaca mulatta (Malpeli and Baker, 1975). Assuming near equivalence between man and the macaque in this regard, it is possible to explain the peculiar distribution of the visual field defects in our patients in the following way.

The shape of the LGN is reminiscent of a kidney, with a hilum on the lower medial aspect (Fig. 6). Most of the optic tract fibres enter the 


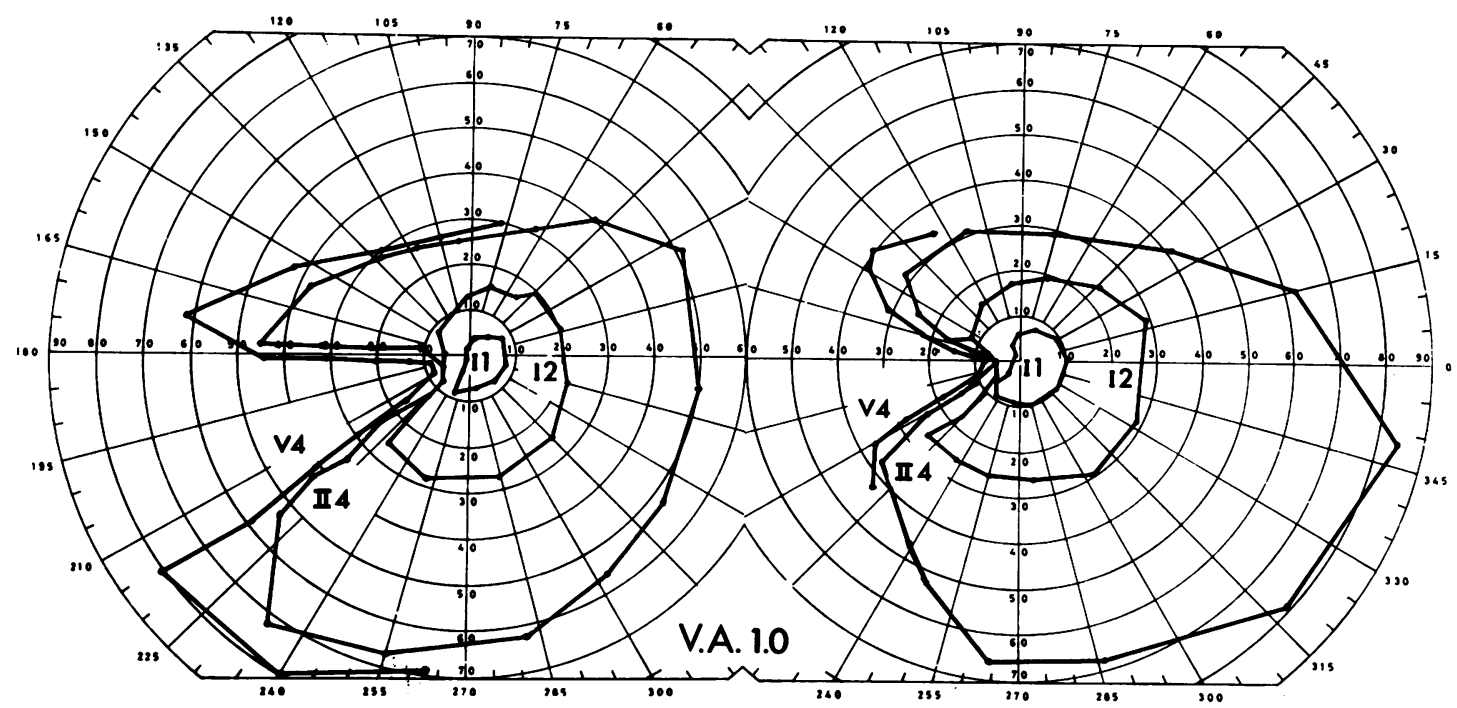

Fig. 4 Case 2: visual field defects.

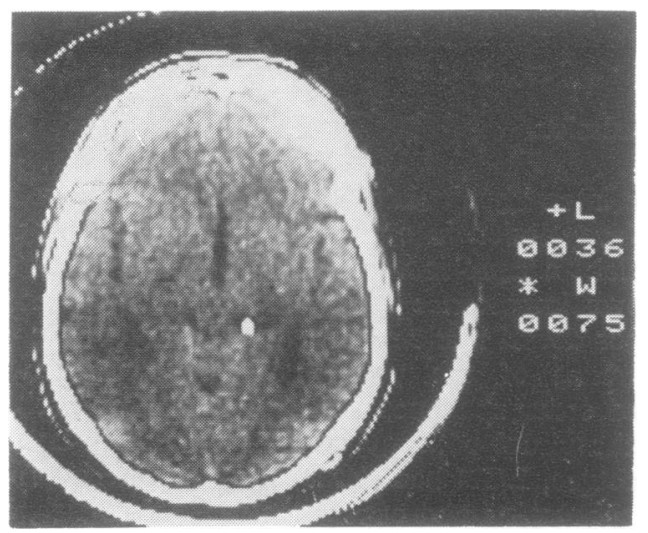

Fig. 5 Case 2: computerised axial tomogram at the level of the lateral geniculate bodies. Note tip of ventricular catheter in ambient cistern on the right and adjacent, rounded area of abnormally low attenuation. The latter represents the site of the visual pathway lesion (see text).

LGN anteriorly, on its convex surface, and the optic radiation leaves it superoposteriorly. The separate projection of nerve fibres from the two eyes upon the LGN explains its characteristic lamination: grey layers of cell bodies alternate with lighter bands of medullated nerve fibres. Fibres from the ipsilateral eye end in cell layers 2,3 , and 5 , and fibres from the contralateral eye end in layers 1, 4, and 6. Corresponding points in the retinae project in register in the $L G N$, so that each point in the visual field is represented by a projection column in the nucleus. Projection columns fan out from the hilum and intersect with the convex LGN surface more or less perpendicularly. Each line in the visual field is, therefore, represented by a set of adjacent columns, or a band, in the nucleus. The horizontal meridian of the visual field, for instance, occupies a somewhat wavy band running along the long axis of the nucleus (interrupted lines in Fig. 6). This band is inclined about 45 degrees of angle with respect to the sagittal plane. The fovea occupies the posterior pole of the LGN. The vertical meridian is represented in a roughly $C$ shaped band centred on the posterior pole; the band extends forwards along the periphery of the nucleus. The upper quadrant of the visual field is represented anterolaterally in the LGN, the lower quadrant anteromedially. The scale of representation diminishes with increasing eccentricity, as indicated along the lower oblique meridian in Fig. 6 (dotted line).

An absolute defect of the visual field presumably requires a full thickness lesion of the nucleus, along its projection columns. The type of visual field defect encountered in case 1 (Fig. 2), therefore, requires a full thickness lesion of the posterior pole and part of the medial wing of the right LGN. The presumed extent of damage is indicated by the dotted area in the 


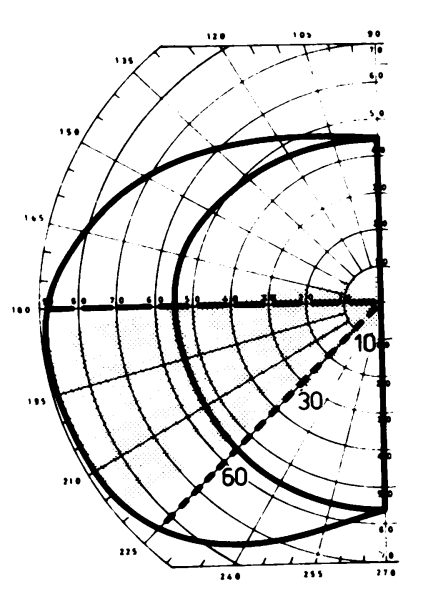

- horizontal meridian of visual field

.....- lower oblique meridian of visual field

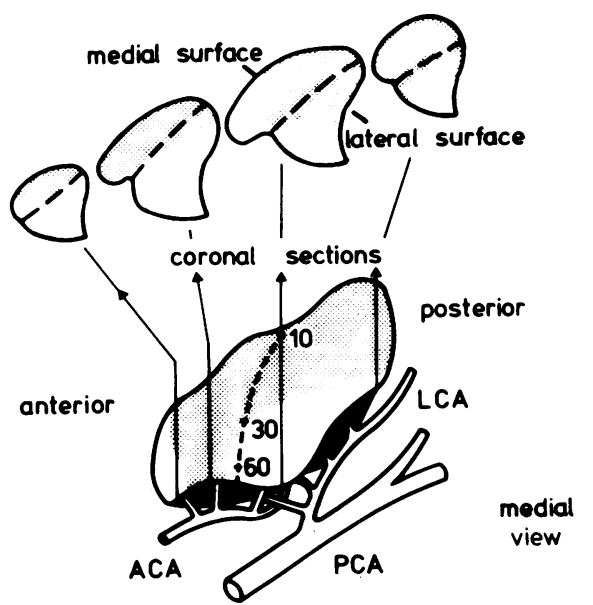

territory of ACA
Fig. 6 Schematic diagram of right lateral geniculate body and its binocular visual field. Shaded areas represent presumed extent of a lesion producing homonymous, sectorial anopia. $A C A$ identifies the anterior choroidal artery, LCA the lateral choroidal artery, and PCA the posterior cerebral artery. schematic diagram (Fig. 6), which is based on the macaque data furnished by Malpeli and Baker (1975). Our second patient must have had a smaller posterior extent of his lesion because of the sparing of the central visual field (Fig. 4).

It is difficult to conceive of any alternative to a vascular lesion in our two patients, with their sudden onset of symptoms, their stationary defects, and the abruptly sloping borders of the visual field defects. An examination of the vascular supply of the LGN is, therefore, appropriate. This has been studied in human brains, using a variety of techniques (Abbie, 1933; François et al., 1956; Galloway and Greitz, 1960; Lazorthes, 1961; Fujino, 1962, 1965; Hoyt et al., 1974). The main arteries involved are the anterior choroidal artery (ACA), approaching the LGN from in front, along the optic tract, and the lateral choroidal artery (LCA), passing below the LGN on its way towards the lateral ventricle. The two arteries send perforating twigs and terminal branches to the LGN, more or less in parallel with its projection columns; they do not anastomose within the nucleus. The ACA branches usually supply the anterior hilum and the anterior and lateral aspects of the LGN, while the remainder of the nucleus is supplied by the LCA. This arrangement is represented schematically in Fig. 6. It appears reasonable to infer that ischaemia within the LCA territory of the LGN could result in the visual field defects encountered in our patients. The combination with sectorial optic atrophy is an expected consequence of damage to second order visual pathway neurones (see Hoyt (1976) and Lundström (1977) for recent reviews).

The lateral choroidal artery (or, sometimes, arteries) usually branches off the posterior cerebral artery within its ambient segment (Galloway and Greitz, 1960; Hoyt et al., 1974). It is destined for the trigonal portion of the choroid plexus of the lateral ventricle, which it reaches via a tortuous course along the thalamic-mesencephalic border zone. En route, perforating twigs are given off to the fornix, the posterior pulvinar, the dorsomedial thalamic nucleus, and the LGN. In addition to visual field defects, an occlusion of the LCA stem conceivably could produce memory defects and components of a posterolateral thalamic syndrome-for example, contralateral dysaesthesia. Such changes were not encountered in our patients; case 2 had a memory deficit, but this was of longer standing.

Occlusion of the ACA stem may or may not produce extensive visual field defects, presumably depending on the quality of collateral supply (Morello and Cooper, 1955). The anterior choroidal artery constitutes a main supply of the optic tract, so any visual field defects associated with its occlusion could be explained on a tract level. Although distal occlusion of the ACA branches to the LGN certainly may occur, as proved by the necropsy study of Mackenzie et al. (1933), clinical recognition of such a lesion appears not to have been reported. On the whole, LGN lesions are much more frequently diagnosed 
at necropsy than during life (Lindenberg and Walsh, 1964; Lindenberg et al., 1973).

Gunderson and Hoyt (1971), and Hoyt (1975), have emphasised incongruous hemianopia ("geniculate hemianopia") as a hallmark of LGN damage. In our view, this is an oversimplification. It is true that the intricate retinotopic organisation of the LGN indicates that many different types of visual field defects (including relative defects, incongruous defects, and monocular defects) may be produced by pathological processes that can encroach upon the nucleus from different directions. Vascular lesions, however, are bound to respect the anatomical boundaries created by the intricately organised vascular supply of the nucleus. Vascular lesions should, therefore, be prone to produce absolute and congruous visual field defects, in particular homonymous, congruous, sectorial defects with abruptly sloping borders. In our view, only disease processes respecting anatomical boundaries within the LGN can be expected to produce diagnostic combinations of symptoms and signs. Homonymous, horizontal sectoranopia, and sectorial optic atrophy, represent such a combination, compatible with ischaemia in the territory of the lateral choroidal artery.

\section{References}

Abbie, A. A. (1933). The blood supply of the lateral geniculate body, with a note on the morphology of the choroidal arteries. Journal of Anatomy, 67, 491521 .

Cogan, D. G. (1945). Syndrome of nonsyphilitic interstitial keratitis and vestibuloauditory symptoms. Archives of Ophthalmology, 33, 144-150.

François, J., Neetens, A., and Collette, J. M. (1956). Vascularization of the optic pathway. IV. Optic tract and external geniculate body. British Journal of Ophthalmology, 40, 341-354.

Fujino, T. (1962). The blood supply of the lateral geniculate body. Japanese Journal of Ophthalmology, 6, 24-33.

Fujino, T. (1965). The intrastitial blood supply of the lateral geniculate body. Archives of Ophthalmology, 74, 815-821.

Galloway, I. R., and Greitz, T. (1960). The medial and lateral choroid arteries. An anatomic and roentgenographic study. Acta Radiologica, 53, 353-366.

Gunderson, C. H., and Hoyt, W. F. (1971). Geniculate hemianopia: incongruous homonymous field defects in two patients with partial lesions of the lateral geniculate nucleus. Journal of Neurology, Neurosurgery, and Psychiatry, 34, 1-6.
Henschen, S. E. (1898). Ueber Localisation innerhalb der äusseren Knieganglions. Neurologisches Centralblatt, 17, 194-199.

Hoyt, W. F. (1975). Geniculate hemianopias: incongruous visual defects from partial involvement of the lateral geniculate nucleus. Proceedings of the A ustralian Association of Neurologists, 12, 7-16.

Hoyt, W. F. (1976). Ophthalmoscopy of the retinal nerve fibre layer in neuro-ophthalmologic diagnosis. Australian Journal of Ophthalmology, 14, 14-34.

Hoyt, W. F., and Kommerell, G. (1973). Der Fundus oculi bei homonymer Hemianopsie. Klinische Monatsblätter der A ugenheilkunde, 162, 456-464.

Hoyt, W. F., Newton, T. H., and Margolis, M. T. (1974). The posterior cerebral artery. In Radiology of the Skull and Brain, volume 2, book 2, chapter 65. Edited by T. H. Newton and D. G. Potts. C. V. Mosby: St Louis.

Kupfer, C. (1962). The projection of the macula in the lateral geniculate nucleus of man. American Journal of Ophthalmology, 54, 597-609.

Lazorthes, G. (1961). Vascularization et Circulation Cérébrale, pp. 122-123. Masson: Paris.

Lindenberg, R., and Walsh, F. B. (1964). Vascular compressions involving intracranial visual pathways. Transactions of the American Academy of Ophthalmology and Otolaryngology, 68, 677-694.

Lindenberg, R., Walsh, F. B., and Sacks, J. G. (1973). Neuropathology of Vision: an Atlas, pp. 335-361. Lea and Febiger: Philadelphia.

Lundström, M. (1977). Optic atrophy in compression of the chiasm. A funduscopic study of the human retinal nerve fibre layer, p. 56. Thesis: University of Göteborg.

Mackenzie, I., Meighan, S., and Pollock, E. N. (1933). On the projection of the retinal quadrants on the lateral geniculate bodies, and the relationship of the quadrants to the optic radiations. Transactions of the Ophthalmological Society of the United Kingdom, 53, 142-169.

Malpeli, J. G., and Baker, F. H. (1975). The representation of the visual field in the lateral geniculate nucleus of Macaca mulatta. Journal of Comparative Neurology, 161, 569-594.

Morello, A., and Cooper, I. S. (1955). Visual field studies following occlusion of the anterior choroidal artery. American Journal of Ophthalmology, 40, 796-801.

Smith, R. S. (1970). Horizontal sector hemianopia of non-traumatic origin. British Journal of Ophthalmology, 54, 208-210.

Spalding, J. M. K. (1952). Wounds of the visual pathway. Part 1: the visual radiation. Journal of Neurology, Neurosurgery, and Psychiatry, 15, 99109.

Spear, P. D., Smith, D. C., and Williams, L. L. (1977). Visual receptive-field properties of single neurons in cat's ventral lateral geniculate nucleus. Journal of Comparative Neurology, 40, 390-409. 